\title{
Binding interactions of perfluoroalkyl substances with thyroid hormone transport proteins and potential toxicological implications
}

\author{
Xiao-Min Ren ${ }^{a}$, Wei-Ping Qin ${ }^{a}$, Lin-Ying Cao ${ }^{a}$, Jing Zhang ${ }^{a}$, Yu Yang, Bin Wan ${ }^{a}$, \\ Liang-Hong Guo ${ }^{\mathrm{a}, \mathrm{b}, *}$ \\ a State Key Laboratory of Environmental Chemistry and Eco-toxicology, Research Center for Eco-environmental Sciences, Chinese Academy of Sciences, 18 \\ Shuangqing Road, Beijing 100085, China \\ ${ }^{\mathrm{b}}$ Institute of Environment and Health, Jianghan University, Wuhan 430056, China
}

\section{A R T I C L E I N F O}

\section{Article history:}

Received 2 June 2016

Received in revised form 11 August 2016

Accepted 11 August 2016

Available online 12 August 2016

\section{Keywords:}

Perfluoroalkyl substances

Transthyretin

Thyroxine-binding globulin

Binding interaction

\begin{abstract}
A B S T R A C T
Perfluoroalkyl substances (PFASs) have been shown to cause abnormal levels of thyroid hormones (THs) in experimental animals, but the molecular mechanism is poorly understood. Here, a fluorescence displacement assay was used to determine the binding affinities of 16 PFASs with two major TH transport proteins, transthyretin (TTR) and thyroxine-binding globulin (TBG). Most of the tested PFASs bound TTR with relative potency (RP) values of $3 \times 10^{-4}$ to 0.24 when compared with that of the natural ligand thyroxine, whereas fluorotelomer alcohols did not bind. Only perfluorotridecanoic acid and perfluorotetradecanoic acid bound TBG, with RP values of $2 \times 10^{-4}$ when compared with that of thyroxine. Based on these results, it was estimated that displacement of T4 from TTR by perfluorooctane sulfonate and perfluorooctanoic acids would be significant for the occupationally exposed workers but not the general population. Structure-binding analysis revealed that PFASs with a medium chain length and a sulfonate acid group are optimal for TTR binding, and PFASs with lengths longer than 12 carbons are optimal for TBG binding. Three mutant proteins were prepared to examine crucial residues involved in the binding of PFASs to TH transport proteins. TTR with a K15G mutation and TBG with either a R378G or R381G mutation showed decreased binding affinity to PFASs, indicating that these residues play key roles in the interaction with the compounds. Molecular docking showed that the PFASs bind to TTR with their acid group forming a hydrogen bond with $\mathrm{K} 15$ and the hydrophobic chain towards the interior. PFASs were modeled to bind TBG with their acid group forming a hydrogen bond with R381 and the hydrophobic chain extending towards R378. The findings aid our understanding of the behavior and toxicity of PFASs on the thyroid hormone system.
\end{abstract}

(c) 2016 Elsevier Ireland Ltd. All rights reserved.

\section{Introduction}

Perfluoroalkyl substances (PFASs) are a class of organic chemicals that have all hydrogen atoms of the hydrocarbon backbone substituted by fluorine atoms (Lau, 2012a). Over the past few decades, PFASs have been widely used in a variety of industrial and commercial applications, such as cooking utensils, food packaging, and water- and stain-resistant materials (Lindstrom et al., 2011). Consequently, PFASs have been widely and frequently detected in wildlife and humans, with perfluorooctane sulfonate

\footnotetext{
Abbreviations: K, lysine; G, glycine; R, arginine.

* Corresponding author at: State Key Laboratory of Environmental Chemistry and Eco-toxicology, Research Center for Eco-environmental Sciences, Chinese Academy of Sciences, 18 Shuangqing Road, Beijing 100085, China.

E-mail address: LHGuo@rcees.ac.cn (L.-H. Guo).
}

(PFOS) and perfluorooctanoic acid (PFOA) having the highest concentrations (Calafat et al., 2007; Lau 2012b; Olsen et al., 2007).

In 2000, 3M, the major manufacturer of PFOS in the USA, announced the phase out of its production of PFOS. Additionally, the US Environmental Protection Agency initiated the PFOA Stewardship Program with industry as a scheme to eliminate emissions and product content of these chemicals by 2015 (EPA, 2011, 2013). Thus, a downward trend for PFOS and PFOA in the general population of USA has been noted (Kato et al., 2011). However, there are still other producers of PFOS-related compounds around the world, which likely maintain the unfavorable global emission levels. Moreover, increasing use of alternative shorter carbon-chain PFASs, such as perfluorobutane sulfonate (PFBS), perfluorohexanoic acid (PFHXA) and perfluorohexane sulfonate (PFHXS), has resulted in increasing concentrations of these PFASs in the environment and humans (Kato et al., 2011). In 
addition, perfluortelomer alcohols such as 8:2 fluorotelomer alcohol (FTOH-8:2) degrade to PFOA and possibly perfluorononanoic acid (PFNA) in biological systems (Martin et al., 2005), which may also contribute to exposure of PFASs to humans. Because of this persistent and widespread exposure to PFASs, there is significant concern about the potential harmful environment and health effects associated with PFASs use.

Extensive laboratory studies have demonstrated that PFASs exposure has been associated with many negative effects, including endocrine disruption, neurotoxicity, disruption to fetal development, and possible carcinogenic effects (Lau et al., 2007). In recent years, there is a growing concern about the disruption effect of PFASs to the thyroid hormone (TH) system (Berg et al., 2015; Webster et al., 2014). Numerous animal studies have suggested that exposure to PFASs may impair thyroid function (Liu et al., 2011; Yu et al., 2009). Langley and Pilcher (1985) and Gutshall et al. (1988) initially reported that a single dose of perfluorondecanoic acid (PFDA) significantly reduced THs levels, lowered body temperature, and depressed heart rate in rats. Several studies have also shown a depression of serum THs levels in PFOS-exposed rats (Lau et al., 2003; Seacat et al., 2003; Thibodeaux et al., 2003). Furthermore, some human epidemiologic studies have indicated the potential disruptive effects of PFASs on human thyroid function (Dallaire et al., 2009). For example, the results of a large-scale survey of the general adult population of the USA have indicated that higher concentrations of serum PFOA and PFOS are associated with current thyroid disease (Melzer et al., 2010). Lopez-Espinosa et al. (2012) suggested associations of serum PFOS with increase of total T4 level and of serum PFOA with hypothyroidism in the children living near a chemical plant located in the USA.

THs play important roles in many biological processes, including modulation of growth, differentiation of many organs, energy homeostasis, and numerous key metabolic pathways (Brent, 2012). The THs, including triiodothyronine (T3) and thyroxine (T4), are produced by the thyroid gland and transported in human plasma by primarily binding to two proteins, transthyretin (TTR) and thyroxine-binding globulin (TBG), responsible for $20 \%$ and $75 \%$ binding activity, respectively. After getting into the target cells, the THs exert their function via binding to the $\mathrm{TH}$ receptors (Yen, 2001). Thus, there are at least four different levels at which chemicals may interfere with thyroid homeostasis: at the receptor level, in THs biosynthesis, in THs metabolism, and in THs transport in the bloodstream (Gutshall et al., 1989; Ren et al., 2015). Competitive binding of chemicals with transport proteins might displace THs from the proteins and disturb the normal levels of THs in the blood stream. Some drugs such as diethylstilbestrol and some pollutants such as hydroxylated polybrominated diphenyl ethers (OH-PBDEs) and polychlorinated biphenyl have been reported to possibly disrupt the thyroid functions by competitive binding to TTR or TBG (Cao et al., 2010; Liu et al., 2012; Ren and Guo 2012; Ucan-Marin et al., 2010). Gutshall et al. (1989) examined the displacement of radio-labeled T4 from rat albumin in vitro by PFDA, suggested that PFDA may disrupt the TH system by displacing circulating hormone from their plasma protein binding sites. Chang et al. (2008) observed a decrease in total T4, a transient increase in free T4, a transient decrease in TSH in circulation, and an increase in urinary excretion of labeled tracer from radio-labeled T4 following a single dose of PFOS, suggesting that PFOS may also act TH system disruption effect by displacing THs from their binding proteins in circulation. Based on the information obtained from previous studies, it is therefore worthwhile to investigate the binding of PFASs with $\mathrm{TH}$ transport proteins as a potential disruption mechanism of thyroid function.
Currently, information about the interaction of PFASs with TH transport proteins is limited, with only one report presenting the binding affinities of PFASs to TTR. Weiss et al. (2009) investigated the binding potencies of 24 PFASs towards human TTR by a radioligand-binding assay. They found the binding potencies of PFASs with TTR were 12.5-50 times lower than the natural ligand thyroxine (T4). Simple descriptors based on the two-dimensional molecular structures of the compounds suggested a dependence on molecular size and functional groups (Kovarich et al., 2012; Weiss et al., 2009). However, the binding mode and mechanism of the structure-dependent binding potency between PFASs and TTR was not revealed in these studies. In addition, the binding potencies of PFASs with TBG, another major TH transport protein, are unknown.

In the present study, the binding of PFASs to human $\mathrm{TH}$ transport proteins (both TTR and TBG) were examined, and critical structural features that define the interactions were characterized. We quantitatively assessed the binding affinities of 16 PFASs to TTR and TBG by a fluorescent competitive binding assay. To identify critical residues involved in the interaction between $\mathrm{TH}$ transport proteins and PFASs, we prepared three mutants and compared the binding potencies of PFASs with these mutants. Furthermore, molecular docking was used to simulate the interactions of these compounds with TH transport proteins in an effort to understand the structural basis of the determined PFASs-binding activities.

\section{Materials and methods}

\subsection{Chemicals}

Human wild-type TTR and TBG were purchased from Calbiochem (San Diego, CA, USA). Human TTR mutant [TTR with substitution of K15 with glycine, TTRmutK15G] and the two human TBG mutants [TBG with substitution of R378 with glycine, TBGmutR378G and TBG with substitution of R381 with glycine, TBGmutR381G] were prepared by Zhongding Biotechnology Co. Ltd. (Nanjing, China). T4 was purchased from Sigma-Aldrich (St. Louis, MO, USA). The fluorescence probe, fluorescein-thyroxine (FT4), was prepared in our laboratory according to a method reported previously (Smith, 1977). The set of PFASs selected for testing was based on environmental relevance and on a broad variation in chemical functionalities, i.e., carbon (C) chain length (from C4 to C14), and different charged end groups (carboxylate, sulfonate, hydroxyl). Sixteen PFASs, including ten perfluoroalkyl acids: perfluorobutyric acid (PFBA), PFHxA, perfluoroheptanoic acid (PFHpA), PFOA, PFNA, PFDA, perfluoroundecanoic acid, (PFUnA), perfluorododecanoic acid (PFDoA), perfluorotridecanoic acid (PFTA), and perfluorotetradecanoic acid (PFTdA); three perfluoroalkyl sulfonates: PFBS, PFHxS, and PFOS; and three perfluorotelomer alcohols: 6:2-, 8:2-, and 10:2- perfluorotelomer alcohol (FTOH-6:2, FTOH-8:2, FTOH-10:2) were used in the experiments (Fig. 1). PFOS was purchased from Alfa Aesar (Ward Hill, MA, USA). The remaining 15 compounds were purchased from Sigma-Aldrich (St. Louis, MO, USA). All 16 PFASs were dissolved in DMSO with concentrations of 20 or $50 \mathrm{mM}$. All other reagents were of the highest quality available.

\subsection{Spectroscopic measurements}

Steady-state fluorescence was measured on a Horiba Fluoromax-4 spectrofluorometer (Edison, NJ, USA). For polarization measurements, the fluorescent molecule was excited with vertically polarized $485 \mathrm{~nm}$ light, and its vertical (I\|) and horizontal $(\mathrm{I} \perp$ ) fluorescence emission intensities at $520 \mathrm{~nm}$ were measured. The polarization value, indicated in millipolarization 
<smiles>NC(Cc1cc(I)c(Oc2cc(I)c(O)c(I)c2)c(I)c1)C(=O)O</smiles>

T4<smiles>O=S(=O)(O)C(F)(F)C(F)(F)C(F)(F)F</smiles>

perfluorinated alkyl sulfonate

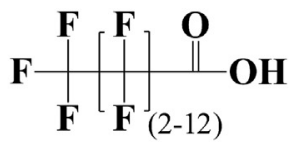

perfluorinated alkyl acids

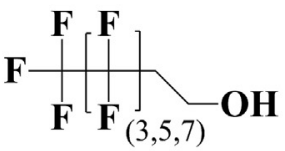

perfluorinated telomer alcohol

Fig. 1. Structures of the compounds used in the experiments.

units ( $\mathrm{mP}$ ), was calculated according to the following equation (Zheng et al., 2008):

$\mathrm{mP}=10^{3} \times(\mathrm{I} \|-\mathrm{I} \perp) /(\mathrm{I} \|+\mathrm{I} \perp)$.

Circular dichroism (CD) spectra were recorded by a JASCO J-815 spectropolarimeter (JASCO Inc., Tokyo, Japan) using a 1-mm cuvette (Hellma, Müllheim, Germany). Spectra were recorded with a $2-\mathrm{nm}$ bandwidth, a $50-\mathrm{nm} / \mathrm{min}$ scan rate, and 1 -s time constant.

\subsection{Direct fluorescent ligand binding assay}

Direct fluorescent ligand binding measurements were performed to measure the dissociation constants of F-T4 ( $\left.K_{\mathrm{d} \text {,probe }}\right)$ with wild-type and mutant TH transport proteins. For TTR and TTRmutK15G, various concentrations of the proteins (5-2000 nM) were added to $50 \mathrm{nM} \mathrm{F-T4}$ in a total volume of $100 \mu \mathrm{L}$. For TBG, TBGmutR378G and TBGmutR381G, various concentrations of the proteins (5-1000 nM) were added to $50 \mathrm{nM} \mathrm{F-T4} \mathrm{in} \mathrm{a} \mathrm{total} \mathrm{volume}$ of $100 \mu \mathrm{L}$. After standing for $5 \mathrm{~min}$ at room temperature, each sample was subjected to a fluorescence polarization measurement. The polarization value at $520 \mathrm{~nm}$ was plotted as a function of protein concentration. The $K_{\mathrm{d} \text {,probe }}$ values of F-T4 with wild-type and mutant $\mathrm{TH}$ transport proteins were then calculated by nonlinear regression curve fitting of the binding data using the Graphpad Prism software (La Jolla, CA, USA).

\subsection{Competitive binding assay}

To determine the binding affinities of PFASs with wild-type and mutant TH transport proteins, a fluorescence polarization-based competitive binding assay with the F-T4 probe was used. For the TTR (TTRmutK15G) competitive binding assay, $50 \mathrm{nM}$ TTR (or $500 \mathrm{nM}$ TTRmutK15G), $50 \mathrm{nM}$ F-T4, and different concentrations of the ligands were mixed in a total volume of $100 \mu \mathrm{L}$, and incubated for $5 \mathrm{~min}$ at room temperature. For the TBG (TBGmutR378G or TBGmutR381G) competitive binding assay, 50 nM TBG (or $1000 \mathrm{nM}$

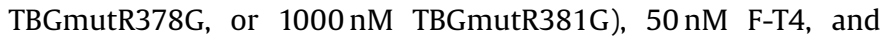
different concentrations of the ligands were mixed in a total volume of $100 \mu \mathrm{L}$, and incubated for $5 \mathrm{~min}$ at room temperature. The fluorescence polarization at $520 \mathrm{~nm}$ was then measured and plotted as a function of ligand concentration. The content of DMSO in the final solution was kept below $1 \%$ to avoid the solvent effect. The competition curves were fitted with a sigmoidal model (OriginLab, Northampton, MA, USA) to derive the $\mathrm{IC}_{50}$ value (the concentration of the ligand required to displace half of the probe from the protein). Relative potency (RP) in comparison with $\mathrm{T} 4$ was obtained by dividing the $\mathrm{IC}_{50}$ of $\mathrm{T} 4$ by that of the $\mathrm{IC}_{50}$ value of the chemical. The dissociation constants of the examined compounds $\left(K_{\mathrm{d}, \text { ligand }}\right)$ with the $\mathrm{TH}$ transport proteins were then calculated according to the following equation:

$\mathrm{IC}_{50} /[\mathrm{F}-\mathrm{T} 4]=K_{\mathrm{d}, \text { ligand }} / K_{\mathrm{d} \text {,probe, }}$

where [F-T4] is the concentration of F-T4.

\subsection{Molecular docking}

AutoDock 4.2 (La Jolla, CA, USA) was used to find the binding mode of PFASs to wild-type TTR and TBG. We extracted the crystal structure of TTR (TTR in complex with T4; PDB ID: 1ICT) and TBG (TBG in complex with T4; PDB ID: 2RIW) from the RCSB Protein Data Bank (http://www.rcsb.org/pdb). The protein files were prepared by removal of the water molecules and other ligands, addition of polar hydrogens, and Kollman charges. The threedimensional coordinates of the ligands in the PDB format were obtained through the PRODRG server using the structure sketch drawing tool (Schüttelkopf and van Aalten, 2004). When docking, the proteins were held rigid, while all torsional bonds of each ligand were set free. A pre-calculated three-dimensional energy grid of equally spaced discrete points was generated prior to docking for a rapid energy evaluation, using the program AutoGrid. The grid box, with dimensions of $60 \times 60 \times 60 \AA$, was centered at the T4-binding site of the proteins and covers the entire binding site and its neighboring residues. The distance between two grid points was set to $0.375 \AA$. Docking was performed using the Lamarckian genetic algorithm. For each complex, ten independent docking runs were conducted and the binding mode with the lowest binding energy was chosen.

\subsection{Statistical analysis}

All of the experiments were conducted in triplicate and the data are expressed as the mean values \pm standard deviation $(\mathrm{SD})(n=3)$.

\section{Results}

3.1. Single amino acid mutation had no effect on the structure of $T H$ transport proteins

To identify critical residues of $\mathrm{TH}$ transport proteins involved in the interaction with PFASs, we prepared three mutant proteins (TTRmutK15G, TBGmutR378G, and TBGmutR381G). To investigate 
the effect of single amino acid mutations on the protein structure of TTR and TBG, the solution structure of wild-type TH transport proteins and their mutants were examined using CD spectroscopy. As shown in Fig. 2A, the far-ultraviolet CD spectra of the wild-type TTR exhibited a negative peak at $215 \mathrm{~nm}$. The $C D$ spectrum of the TTRmutK15G mutant exhibited a similar spectrum to that of the wild-type protein, thus showing that the variant retained a similar secondary structure. In addition, the CD spectra of TBGmutR378G and TBGmutR381G mutants exhibited no significant differences when compared with the $\mathrm{CD}$ spectrum recorded of the wild-type protein, showing that these variants retained very similar secondary structures (Fig. 2B). According to the $C D$ results, the single amino acid mutations had negligible effects on the overall structure of the $\mathrm{TH}$ transport proteins.

\subsection{Determination of dissociation constants of F-T4 with wild-type} and mutant $\mathrm{TH}$ transport proteins

To establish a competitive binding assay, the dissociation constants of probe (F-T4) with the target proteins (wild-type and mutant $\mathrm{TH}$ transport proteins) were determined initially. The enhancement of F-T4 fluorescence polarization after binding with a transport protein was used in a direct ligand-binding assay for determining the dissociation constant. For TTR, there are two identical ligand binding sites with different affinities because of negative cooperativity (Neumann et al., 2001). As depicted in
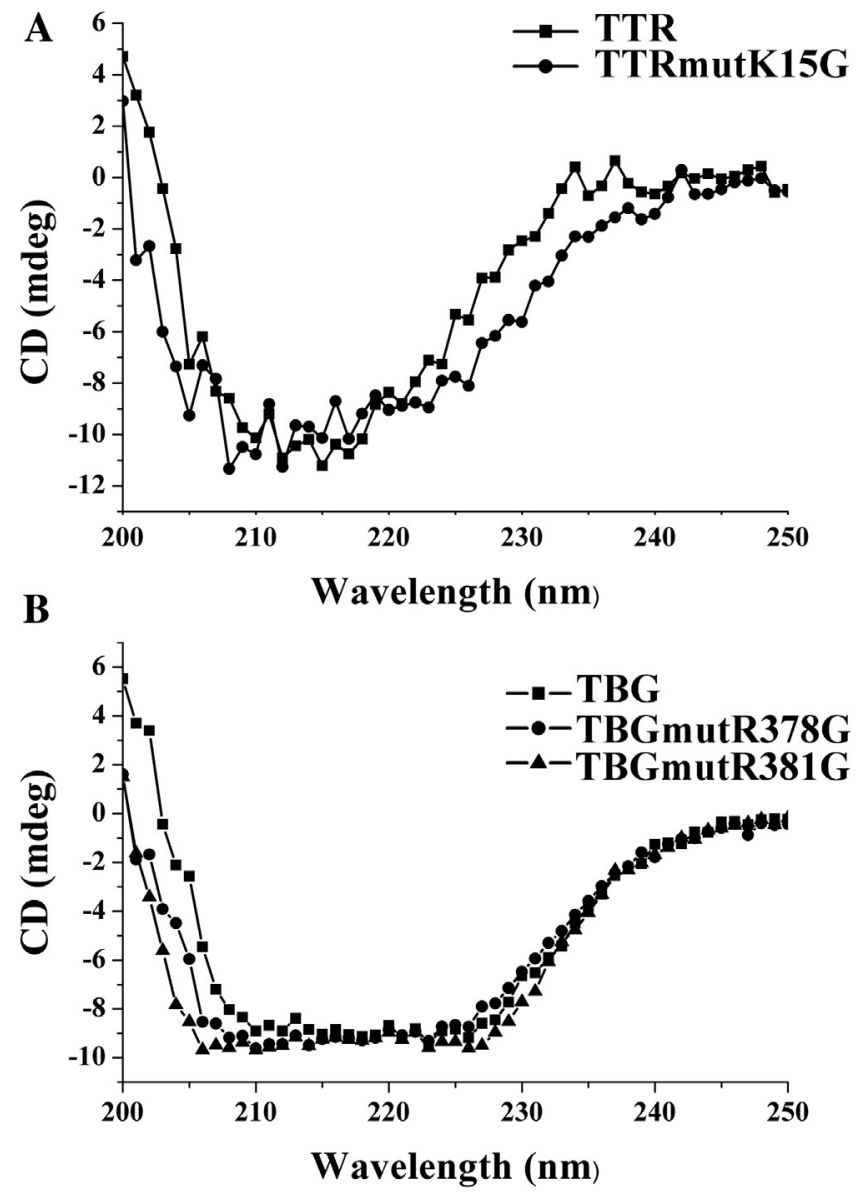

Fig. 2. CD spectra of wild-type and mutant TH transport proteins. (A) CD spectra of $2 \mu \mathrm{M}$ TTR and $2 \mu \mathrm{M}$ TTRmutK15G; (B) CD spectra of $2 \mu \mathrm{M}$ TBG, $2 \mu \mathrm{M}$ TBGmutR378G and $2 \mu \mathrm{M}$ TBGmutR381G.

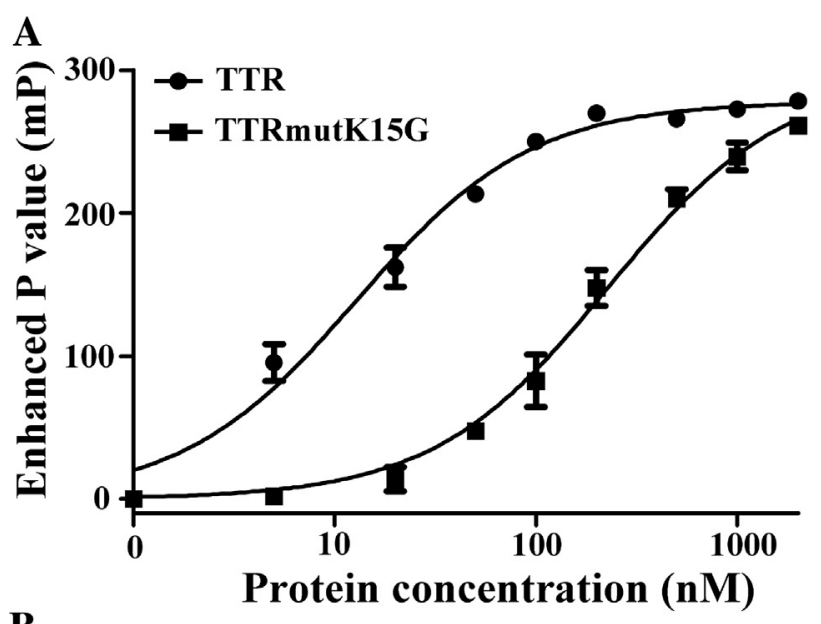

B

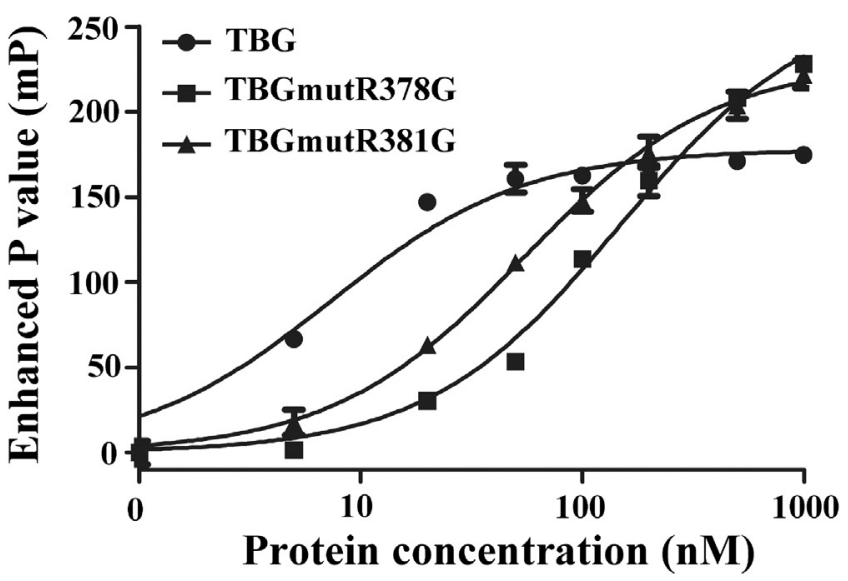

Fig. 3. Direct fluorescence binding curves of F-T4 to wild-type and mutant $\mathrm{TH}$ transport proteins. (A) Fluorescence polarization value of $50 \mathrm{nM}$ F-T4 as a function of added TTR and TTRmutK15G concentration; (B) Fluorescence polarization value of $50 \mathrm{nM} \mathrm{F-T4} \mathrm{as} \mathrm{a} \mathrm{function} \mathrm{of} \mathrm{added} \mathrm{TBG,} \mathrm{TBGmutR378G} \mathrm{and} \mathrm{TBGmutR381G}$ concentration.

Fig. 3A, the fluorescence polarization of F-T4 was enhanced by 270 or $260 \mathrm{mP}$ as the concentration of TTR or TTRmutK15G increased to $2 \mu \mathrm{M}$. The binding data were used to determine $K_{\mathrm{d}}$ values of $8 \mathrm{nM}$ and $27 \mathrm{nM}$ for the two binding sites of TTR $\left(R^{2}=0.98\right)$, and $K_{\mathrm{d}}$ values of 498 and $524 \mathrm{nM}$ were obtained between F-T4 and TTRmutK15G for the two binding sites $\left(R^{2}=0.99\right)$. For TBG, there is only one T4 binding site (Zhou et al., 2006). Similarly, the fluorescence polarization enhancement was 190,260 , and $250 \mathrm{mP}$, as the concentration of TBG, TBGmutR378G, or TBGmutR381G increased to $1 \mu \mathrm{M}$, respectively (Fig. 3B). Using these binding data, $K_{\mathrm{d}}$ values of $7 \mathrm{nM}\left(R^{2}=0.97\right), 148 \mathrm{nM}\left(R^{2}=0.99\right)$, and $54 \mathrm{nM}\left(R^{2}=0.99\right)$ for TBG, TBGmutR378G and TBGmutR381G, respectively, were determined.

3.3. Determination of the binding potencies of PFASs with wild-type and mutant $\mathrm{TH}$ transport proteins

The binding potencies of 16 selected PFASs with wild-type and mutant $\mathrm{TH}$ transport proteins were assessed quantitatively by fluorescence polarization using a competitive binding assay with $\mathrm{T} 4$ as the positive control and reference. The competition curves of all the tested compounds with wild-type and mutant TH transport proteins are shown in Fig. 4 (and Supplementary material, Figs. $\mathrm{S} 1-\mathrm{S} 5)$. From the competition curves, $\mathrm{IC}_{50}, K_{\mathrm{d}}$, and RP values for 
A
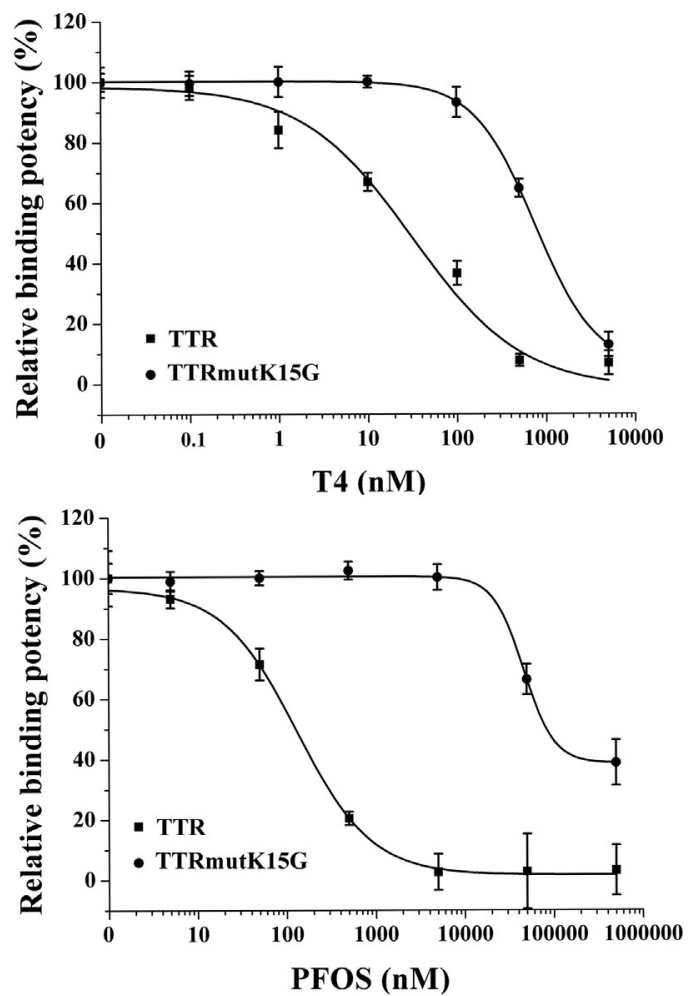

B
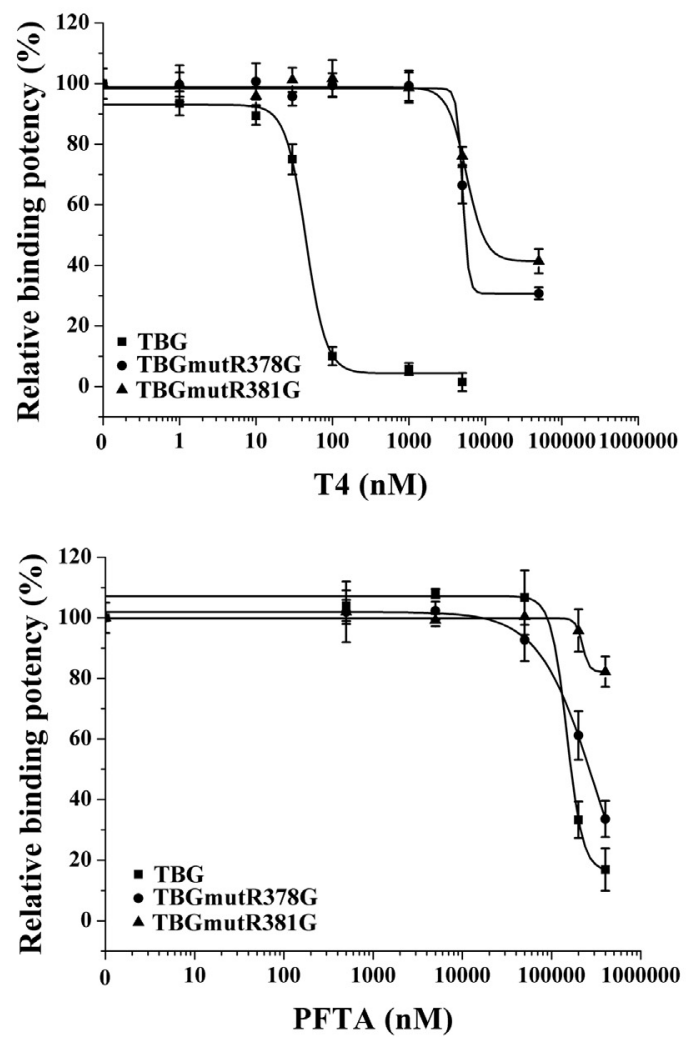
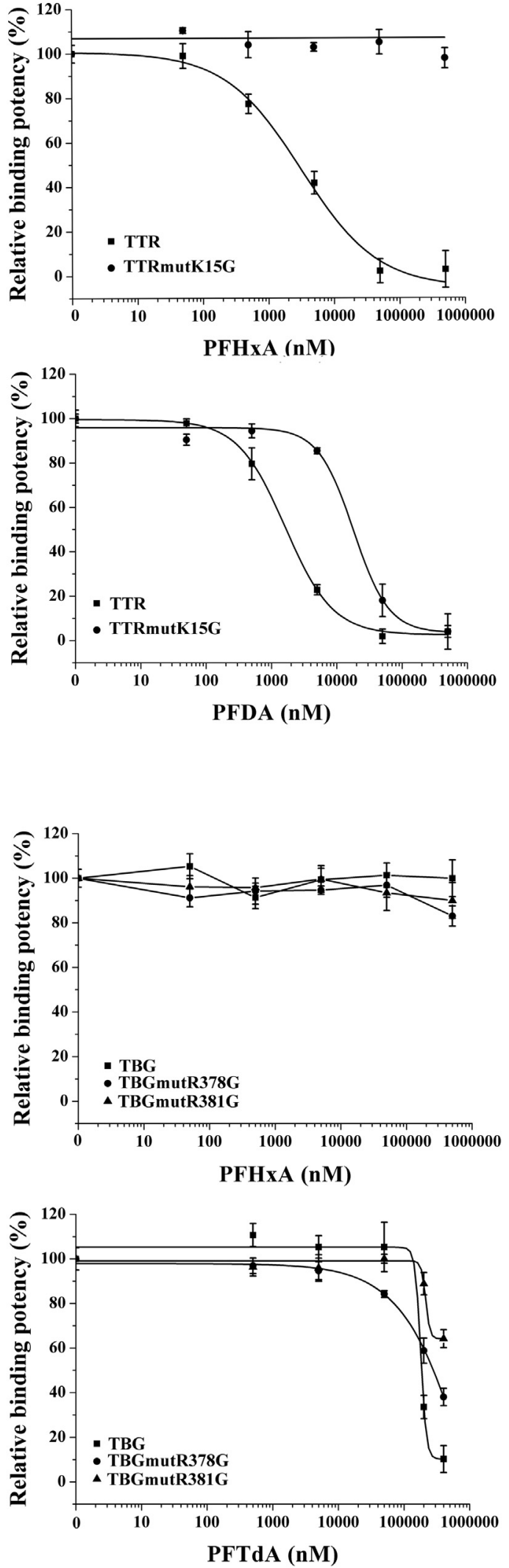

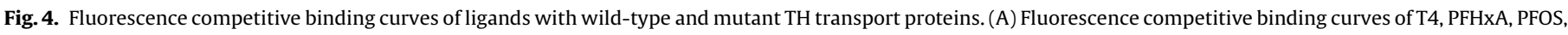

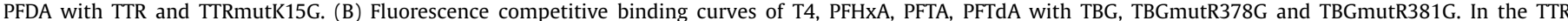

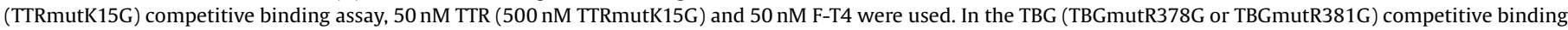

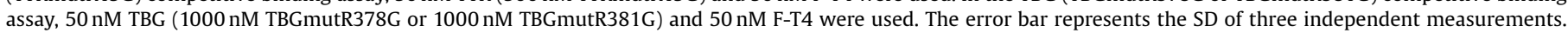


TTR and TBG were calculated and are listed in Tables 1 and 2, respectively.

According to the TTR competitive binding assays, the 16 PFASs showed different binding potencies (Table 1). For the ten perfluoroalkyl acids, the $K_{\mathrm{d}}$ values decreased from $14.3 \mu \mathrm{M}$ to $60 \mathrm{nM}$ as the carbon chain length increased from C4 to C8. However, no further increase, but a downward trend, was observed for perfluoroalkyl acids with longer chain lengths (C9-C14). For the three PFASs, the $K_{\mathrm{d}}$ values decreased from $2.1 \mu \mathrm{M}$ to $20 \mathrm{nM}$ as the carbon chain length increased from C4 to C8. Wild-type TTR showed no binding towards the three fluorotelomer alcohols. In comparison with wild-type TTR, the T4 exhibited much weaker binding affinity to TTRmutK15G. Similarly, the PFASs exhibited much weaker binding affinities to TTRmutK15G compared with wild-type TTR. As shown in Table 1 , the $K_{\mathrm{d}}$ values of TTRmutK15G towards the 10 perfluoroalkyl acids were in the range of ND$182 \mu \mathrm{M}$. For the three PFASs, TTRmutK15G only bound PFOS with a
$K_{\mathrm{d}}$ of $459 \mu \mathrm{M}$. No interaction between TTRmutK15G and the three fluorotelomer alcohols was observed.

According to the TBG competitive binding assays, only PFTA and PFTdA bound to TBG with $K_{\mathrm{d}}$ values of $23 \mu \mathrm{M}$ and $26.6 \mu \mathrm{M}$, respectively (Table 2). Both TBGmutR378G and TBGmutR381G exhibited much weaker binding potencies to PFTA and PFTdA when compared with that of wild-type TBG. TBGmutR378G bound PFTA and PFTdA with $K_{d}$ value of $799 \mu \mathrm{M}$, whereas TBGmutR381G bound PFTA and PFTdA with $K_{\mathrm{d}}$ value of $432 \mu \mathrm{M}$ (Table 2).

\subsection{Molecular docking of PFASs with TH transport proteins}

We used the molecular docking analysis to provide insights into the interactions between PFASs and TH transport proteins. T4 was also included in the docking study. The molecular docking for all tested ligands to TTR and TBG are shown in Fig. 5 (and Supplementary material, Figs. S6 and S7). The results of the

Table 1

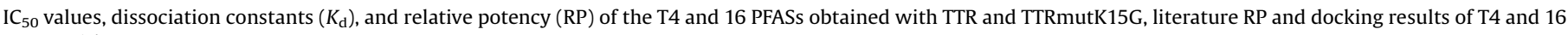
PFASs with TTR.

\begin{tabular}{|c|c|c|c|c|c|c|c|}
\hline \multirow[b]{2}{*}{ Compound } & \multicolumn{4}{|l|}{ TTR } & \multicolumn{3}{|l|}{ TTRmutK15G } \\
\hline & $\mathrm{IC}_{50}(\mathrm{nM})$ & $K_{\mathrm{d}}(\mathrm{nM})$ & $\mathrm{RP}$ & Literature RP & $\mathrm{IC}_{50}(\mathrm{nM})$ & $K_{\mathrm{d}}(\mathrm{nM})$ & Hydrogen bonding \\
\hline $\mathrm{T} 4$ & $31 \pm 14$ & $5 \pm 2$ & 1 & 1 & $751 \pm 33$ & $7479 \pm 328$ & Lys15 \\
\hline PFBA & $89,252^{\mathrm{a}}$ & $14,280^{\mathrm{a}}$ & $3 \times 10-4$ & ND & ND & ND & Lys15 \\
\hline PFHxA & $3189 \pm 1156$ & $510 \pm 184$ & $9 \times 10-3$ & 0.007 & ND & ND & Lys15 \\
\hline PFHрA & $1128 \pm 139$ & $180 \pm 22$ & 0.028 & 0.039 & ND & ND & Lys15 \\
\hline PFOA & $378 \pm 45$ & $60 \pm 7$ & 0.083 & 0.064 & ND & ND & Lys15 \\
\hline PFNA & $1977 \pm 1698$ & $316 \pm 271$ & 0.016 & 0.022 & $60,000^{\mathrm{a}}$ & 597,600 & Lys15 \\
\hline PFDA & $1623 \pm 230$ & $259 \pm 36$ & 0.019 & 0.007 & $18,307^{\mathrm{a}}$ & 182,337 & Lys15 \\
\hline PFUnA & $5339 \pm 300$ & $854 \pm 48$ & 0.006 & 0.003 & $300,000^{a}$ & $2,988,000$ & Lys15 \\
\hline PFDoA & $8082 \pm 5102$ & $1293 \pm 816$ & 0.004 & 0.001 & $100,000^{\mathrm{a}}$ & 996,000 & Lys15 \\
\hline PFTA & $5367 \pm 387$ & $858 \pm 61$ & 0.006 & - & $55,234^{\mathrm{a}}$ & 550,130 & Lys15 \\
\hline PFTdA & $6080 \pm 2112$ & $972 \pm 337$ & 0.005 & 0.002 & $183,000^{\mathrm{a}}$ & $1,822,680$ & Lys15 \\
\hline PFBS & $13,331 \pm 1019$ & $2132 \pm 163$ & 0.002 & 0.003 & ND & ND & Lys15 \\
\hline PFHxS & $594 \pm 63$ & $95 \pm 10$ & 0.053 & 0.085 & ND & ND & Lys15 \\
\hline PFOS & $130 \pm 10$ & $20 \pm 2$ & 0.24 & 0.065 & $46,128 \pm 7032$ & $459,434 \pm 70,038$ & Lys15 \\
\hline FTOH-6:2 & ND & ND & ND & - & ND & ND & \\
\hline FTOH-8:2 & ND & ND & ND & ND & ND & ND & \\
\hline FTOH-10:2 & ND & ND & ND & ND & ND & ND & \\
\hline
\end{tabular}

ND: Not detectable.

-: No report.

${ }^{\mathrm{a}} \mathrm{IC}_{50}$ values were calculated by linear interpolation between two responses located around the $50 \%$ inhibition level.

Table 2

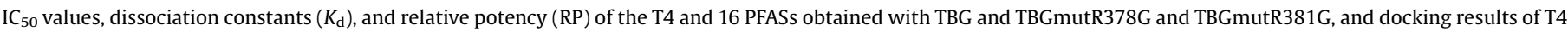
and 16 PFASs with TBG.

\begin{tabular}{|c|c|c|c|c|c|c|c|c|}
\hline \multirow[b]{2}{*}{ Compound } & \multicolumn{3}{|l|}{ TBG } & \multicolumn{2}{|c|}{ TBGmutR378G } & \multicolumn{3}{|c|}{ TBGmutR381G } \\
\hline & $\mathrm{IC}_{50}(\mathrm{nM})$ & $K_{\mathrm{d}}(\mathrm{nM})$ & $\mathrm{RP}$ & $\mathrm{IC}_{50}(\mathrm{nM})$ & $K_{\mathrm{d}}(\mathrm{nM})$ & $\mathrm{IC}_{50}(\mathrm{nM})$ & $K_{\mathrm{d}}(\mathrm{nM})$ & Hydrogen bond \\
\hline $\mathrm{T} 4$ & $44 \pm 5$ & $6 \pm 1$ & 1 & $5500^{\mathrm{a}}$ & $16,280^{a}$ & $8925^{\mathrm{a}}$ & $9639^{a}$ & $\operatorname{Arg} 378$ \\
\hline PFBA & ND & ND & ND & ND & ND & ND & ND & Arg381 \\
\hline PFHxA & ND & ND & ND & ND & ND & ND & ND & Arg381 \\
\hline PFHрA & ND & ND & ND & ND & ND & ND & ND & Arg381 \\
\hline PFOA & ND & ND & ND & ND & ND & ND & ND & Arg381 \\
\hline PFNA & ND & ND & ND & $225,724^{\mathrm{a}}$ & $668,143^{a}$ & $70,000^{a}$ & $75,600^{\mathrm{a}}$ & Arg381 \\
\hline PFDA & ND & ND & ND & $151,663^{\mathrm{a}}$ & $341,178^{a}$ & $500,000^{a}$ & $540,000^{a}$ & Arg381 \\
\hline PFUnA & ND & ND & ND & ND & ND & ND & ND & Arg381 \\
\hline PFDoA & ND & ND & ND & $280,000^{\mathrm{a}}$ & $828,800^{\mathrm{a}}$ & ND & ND & Arg381 \\
\hline PFTA & 170,000 & 23,800 & $2 \times 10-4$ & $270,000^{\mathrm{a}}$ & $799,200^{a}$ & $>400,000$ & $>432,000$ & Arg381 \\
\hline PFTdA & 190,000 & 26,600 & $2 \times 10-4$ & $270,000^{\mathrm{a}}$ & $799,200^{\mathrm{a}}$ & $>400,000$ & $>432,000$ & Arg381 \\
\hline PFBS & ND & ND & ND & ND & ND & ND & ND & Arg381 \\
\hline PFHxS & ND & ND & ND & ND & ND & ND & ND & Arg381 \\
\hline PFOS & ND & ND & ND & ND & ND & ND & ND & Arg381 \\
\hline FTOH-6:2 & ND & ND & ND & ND & ND & ND & ND & \\
\hline FTOH-8:2 & ND & ND & ND & ND & ND & ND & ND & \\
\hline FTOH-10:2 & ND & ND & ND & ND & ND & ND & ND & \\
\hline
\end{tabular}

ND: Not detectable.

${ }^{\mathrm{a}} \mathrm{IC}_{50}$ values were calculated by linear interpolation between two responses located around the $50 \%$ inhibition level. 


\section{A:TTR}

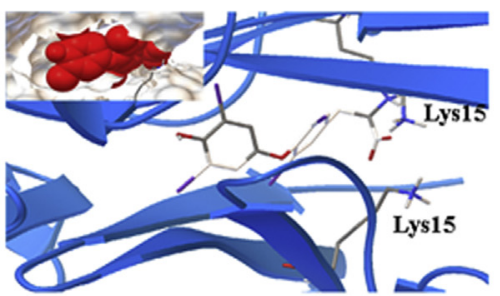

T4

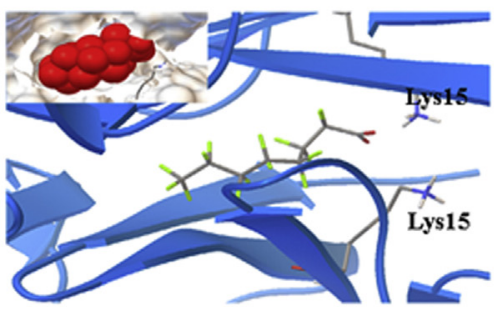

PFOA

\section{B:TBG}

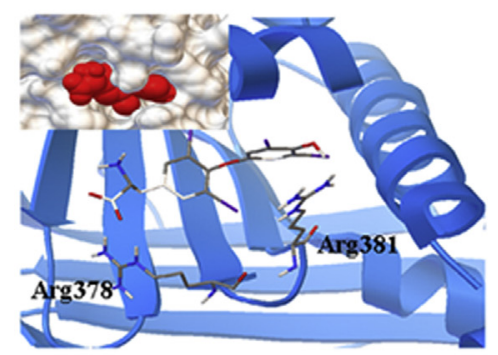

T4

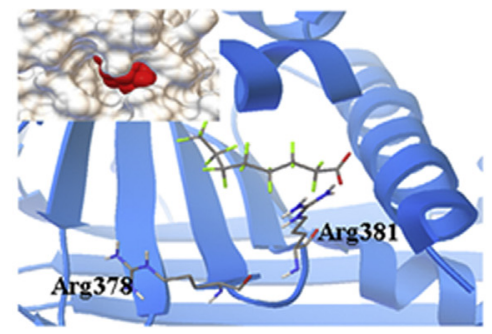

PFOA

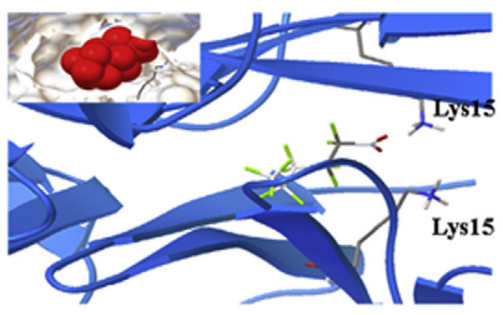

PFHxA

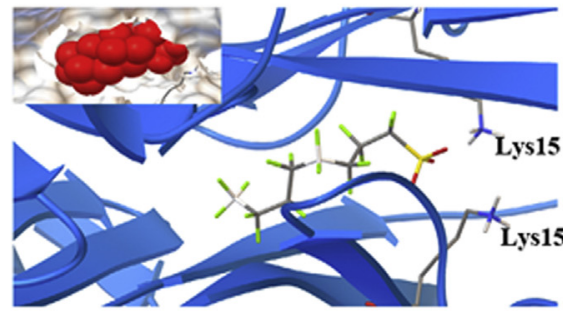

PFOS

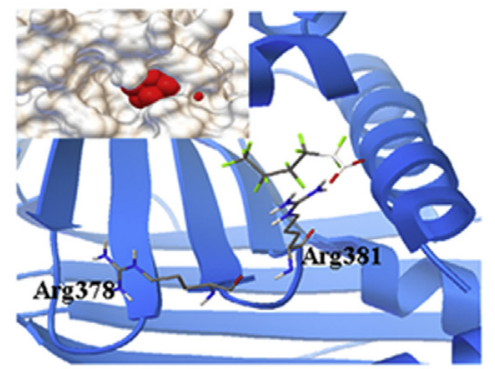

PFHxA

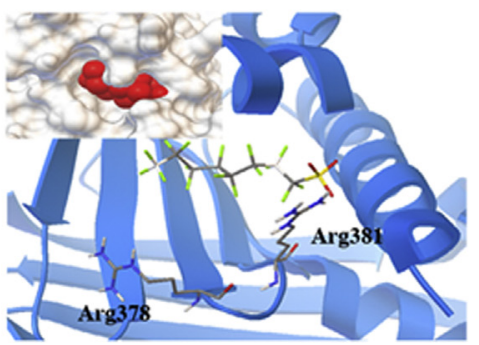

PFOS

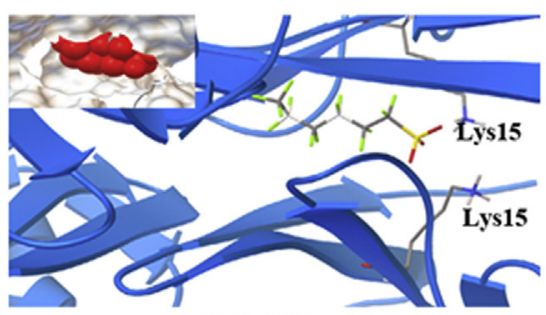

PFHxS

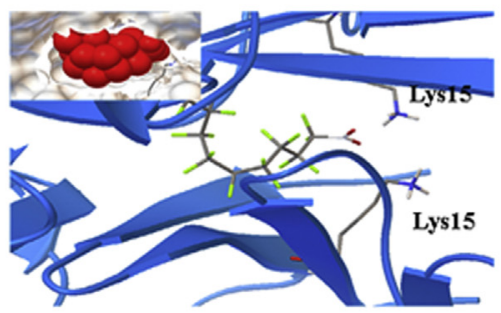

PFDA

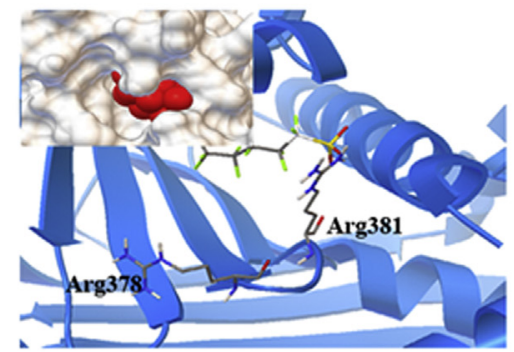

PFHxS

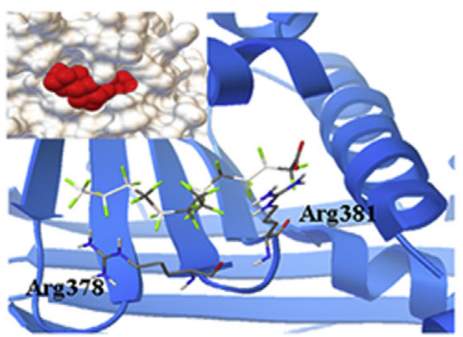

PFTdA

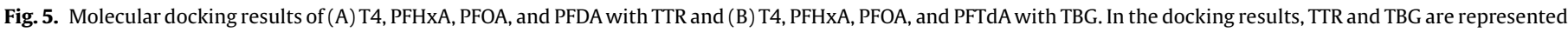

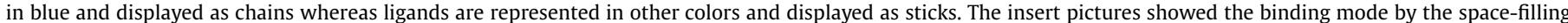

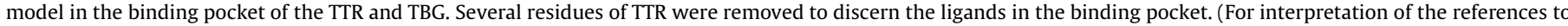
color in this figure legend, the reader is referred to the web version of this article.)

hydrogen bond interactions between the chemicals and TTR and TBG are listed in Tables 1 and 2, respectively.

As shown in Fig. 5A, T4 docked into TTR with the correct orientation in which the $\mathrm{OH}$-phenyl ring remains buried in the interior of the binding pocket and the carboxylate substituent facing towards the entrance of the ligand binding pocket. The hydrogen bond interaction observed between T4 and K15 is consistent with the crystal structure (Wojtczak et al., 2001). For the perfluoroalkyl acids and perfluoroalkyl sulfonates investigated, all compounds could fit into the binding pocket of TTR with similar orientations. The negative charged end group faced towards the entrance of the ligand-binding pocket, and the hydrophobic part towards the interior of the binding site (Fig. 5A). Like T4, 13 PFASs with carboxylate or sulfonate end groups could form a hydrogen bond with K15 (Table 1). No hydrogen bond interaction between TTR and the three fluorotelomer alcohols was observed. Spacefilling models showed that T4 could fill the TTR ligand-binding pocket (Fig. 5A). Similar to T4, PFOA and PFOS could also nearly fill 
the TTR ligand-binding pocket, with the fluorinated carbon tail adopting an extended state (Fig. 5A). However, perfluoroalkyl acids with carbon chain less than $8 \mathrm{C}$ (e.g., PFHxA, PFHxS) did not adequately fill the T4 binding pocket, whereas longer fluorinated carbon chain structures (e.g., PFDA) were nearly too large and required the fluorinated carbon tail to bend so that the ligand could fit into the binding pocket (Fig. 5A).

As shown in Fig. 5B, T4 docked into TBG with the correct orientation in which the carboxylate substituent forms a hydrogen bond with R378 and the OH-phenyl ring directed towards R381 on the other side of the ligand binding pocket. The docking results are consistent with the crystal structure (Qi et al., 2011). Unlike T4 forming a hydrogen bond with R378, the PFASs fit into the binding pocket of TBG with their carboxylate or sulfonate end groups forming a hydrogen bond with R381. The hydrophobic end of PFASs faced towards R378. Space-filling models showed that the perfluoroalkyl acids with carbon chain less than 12C (e.g., PFHxA, PFOA) did not adequately fill the T4 binding pocket, whereas longer fluorinated carbon chain structures (e.g., PFTdA) could nearly fill the TBG ligand-binding pocket (Fig. 5B).

\subsection{Toxicological implications}

With the $K_{\mathrm{d}}$ values of the PFASs with TTR obtained in this study, we can estimate the competitive displacement of TH by PFASs from the TTR. In addition to TTR ( $\sim 0.3 \%$ of total serum proteins), there are many other proteins in human serum, which might also bind with PFASs. The serum includes two major groups of proteins: human serum albumin (HSA) ( $62 \%$ of total serum proteins) and globulin ( $\sim 30 \%$ of total serum proteins). Isolated HSA has been reported to bind with PFASs in our previous study (Chen and Guo, 2009). There are also a few studies on the binding of PFOS and PFOA with proteins in human and animal serum and plasma (Han et al., 2003; Jones et al., 2003). According to their results, albumins are the major binding targets of PFOA and PFOS in serum, with greater than $90 \%$ of the chemicals bound to the proteins. The effect of PFAS binding with other proteins in human serum would be very small and does not need to be considered. Therefore, we consider the binding of PFASs to TTR in the presence of HSA. We made these estimations based on the hypothesis that the concentration of PFASs bound HSA is much less than the concentration of total HSA. Since PFOS and PFOA are two most commonly present PFASs, we estimated the binding of these two PFASs with TTR and HSA. By using a simplified approach, we calculated the concentrations of PFASs bound TTR and PFASs bound HSA, based on the following equations:

$[$ PFAS-T $]+[$ PFAS-H $]+[$ PFAS $]=[$ PFAS $] \mathrm{t}$

$[$ PFAS-T $]+[T]=[T] t$

$[$ PFAS-H $]+[\mathrm{H}]=[\mathrm{H}] \mathrm{t}$

$[\mathrm{PFAS}-\mathrm{T}]=[\mathrm{PFAS}][\mathrm{T}] / K_{\mathrm{d}, \mathrm{TTR}}$

[PFAS-H] $=[\mathrm{PFAS}][\mathrm{H}] / K_{\mathrm{d}, \mathrm{HSA}}$

In the equations, [PFAS-T] is the concentration of TTR bound PFAS, [PFAS-H] is the concentration of HSA bound PFAS, [PFAS] is the concentration of free PFAS, [PFAS]t is the total concentration of PFAS, $[\mathrm{T}]$ is the concentration of free TTR, [T]t is the total concentration of TTR, $[\mathrm{H}]$ is the concentration of free HSA, $[\mathrm{H}] \mathrm{t}$ is the total concentration of HSA, $K_{\mathrm{d}, \mathrm{TTR}}$ is the equilibrium dissociation constant of PFAS with the TTR and $K_{\mathrm{d}, \mathrm{HSA}}$ is the equilibrium dissociation constant of PFAS with the HSA.

The concentration of TTR is about $5.4 \mu \mathrm{M}$ in the normal human serum (Shenkin, 2006). The concentration of HSA is about $600 \mu \mathrm{M}$ in the normal human serum (Schreiber, 2002). The $K_{\mathrm{d}}$ values of PFOS and PFOA with the HSA are $45 \mu \mathrm{M}$ and $3.7 \mu \mathrm{M}$, respectively (Chen and Guo, 2009). The concentration of total T4 in the normal human serum is about $90 \mathrm{nM}$ (Yen, 2001). The concentrations of PFOS and PFOA in the human serum of general population are in the range of $2 \mathrm{nM}-3.3 \mu \mathrm{M}$ and $0.5 \mathrm{nM}-600 \mathrm{nM}$, respectively (Fromme et al., 2009). Serum PFOS and PFOA levels in occupationally exposed workers ranged from $0.6 \mu \mathrm{M}-25.6 \mu \mathrm{M}$ and from less than limit of detection ( $<$ LOD) to $275.6 \mu \mathrm{M}$, respectively (Fromme et al., 2009). Using these values and Eqs. (3)-(7), the concentrations of TTR or HSA bound with T4, PFOS, and PFOA are calculated and listed in Table 3. The calculation shows that, for the general population, chemical-bound TTR concentrations are between $1.9 \mathrm{nM}$ and $2.9 \mu \mathrm{M}$ for PFOS and between $0.2 \mathrm{nM}$ and $207 \mathrm{nM}$ for PFOA. These concentrations are much lower than that of total TTR. There would still be enough free TTR to bind and transport T4 in the serum. However, for occupationally exposed workers, chemical-bound TTR by PFOS and PFOA could reach $5.3 \mu \mathrm{M}$, which is almost the total concentration of TTR in human serum. This suggests that almost all of TTR are occupied by PFASs. Therefore, the competitive displacement of T4 from TTR by PFASs could not be neglected in the occupationally exposed workers. In addition to TTR and HSA, PFASs may bind to some other unknown proteins in human serum, which might decrease the amount of PFASs bind to TTR. Therefore, the fraction of PFASs bound to TTR in our calculation might be overestimated.

\section{Discussion}

By using an F-T4 competitive binding assay, we obtained the binding affinities of 16 PFASs with TTR. As shown in Table 1, with the exception of three fluorotelomer alcohols, all of the tested PFASs bound to wild-type TTR with RP values ranging from $3 \times 10^{-4}$ to 0.24 of T4. In a previous study, Weiss et al. (2009) studied the binding affinities of 24 PFASs with TTR using a radioisotope method. Comparing the results of 14 common PFASs, the RP values obtained in our study are in agreement with those measured previously (Table 1 ). Based on our results as well as those of others, some PFASs possess comparable binding affinities with TTR as T4. According to our simplified estimation, displacement of T4 from TTR by PFASs would be insignificant at the current general population contamination level. However, for the occupationally exposed workers with much higher concentration of PFASs in serum, at least 2 orders of magnitude higher than that of general population, significant displacement of T4 from the TTR would

Table 3

Concentrations of T4, PFOS, and PFOA in human serum and calculated concentrations of T4-bound TTR ${ }^{\mathrm{a}}$, PFOS-bound TTR ${ }^{\mathrm{a}}$, PFOA-bound TTR ${ }^{\mathrm{a}}$, T4-bound HSA, PFOSbound HSA, and PFOA-bound HSA.

\begin{tabular}{llll}
\hline Compound & concentration & chemical-bound TTR & chemical-bound HSA \\
\hline T4 & $\sim 90 \mathrm{nM}$ & $\sim 75 \mathrm{nM}$ & $\sim 15 \mathrm{nM}$ \\
PFOS $^{\mathrm{b}}$ & $2 \mathrm{nM}-3.3 \mu \mathrm{M}$ & $1.9 \mathrm{nM}-2.9 \mu \mathrm{M}$ & $0.1 \mathrm{nM}-310 \mathrm{nM}$ \\
PFOS $^{\mathrm{c}}$ & $0.6 \mu \mathrm{M}-25.6 \mu \mathrm{M}$ & $0.5 \mu \mathrm{M}-5.3 \mu \mathrm{M}$ & $0.1 \mu \mathrm{M}-18.8 \mu \mathrm{M}$ \\
PFOA $^{\mathrm{b}}$ & $0.5 \mathrm{nM}-600 \mathrm{nM}$ & $0.2 \mathrm{nM}-207 \mathrm{nM}$ & $0.3 \mathrm{nM}-390 \mathrm{nM}$ \\
PFOA $^{\mathrm{c}}$ & $<$ LOD-275.6 $\mu \mathrm{M}$ & $<$ LOD-5.2 $\mu \mathrm{M}$ & $<$ LOD-268.5 $\mu \mathrm{M}$ \\
\hline
\end{tabular}

\footnotetext{
All the data are evaluated from the primary binding site.

b General population.

c Occupationally exposed workers.
} 
occur, leading to disruption of $\mathrm{T} 4$ transport. Based on our estimation, PFOS and PFOA could completely displace T4 from TTR in the production workers. The serum TH level in these workers would be very low, and there would be a negative association between PFOS/PFOA with serum TH in this population. However, such association was not observed in the reported occupational bio-monitoring studies (Olsen et al., 2003; Olsen and Zobel, 2007). Since the thyroid homeostasis is complex, there might be other complications which we have not considered yet. Additionally, we provide the first evidence that PFTA and PFTdA could also bind with TBG, another important TH transport protein. Compared with the results of TTR, TBG showed no binding or much weaker binding affinities towards PFASs (Table 2). Since the concentration of TBG $(0.3 \mu \mathrm{M})$ in the normal human serum is much lower than that of TTR $(5.4 \mu \mathrm{M})$ (Shenkin, 2006; Wenzel, 1981), we hypothesize that PFASs might favor binding to TTR over TBG when they enter the human blood stream. With 16 PFASs tested, some structural characteristics of the binding mode with TTR can be analyzed. We found the TTR binding potency is clearly associated with the carbon chain length and the charged end group. PFASs with lengths of $\mathrm{C} 8$ were optimal for TTR binding. TTR binding affinity was also found to be dependent on the charged end group in the order of sulfonate $>$ carboxylate $>$ alcoholic hydroxyl. Our results describing the structure-binding relationship between PFASs and TTR is in good agreement with those of a previous study, which also showed dependencies on the molecular size and functional groups of the compounds tested (Weiss et al., 2009).

To understand the structural basis of the determined PFASbinding activities, we prepared a TTR mutant to identify the critical residue involved in binding PFASs. According to the results of several crystal structure of the TTR-ligands complex, K15 forms a crucial hydrogen bond interaction with T4 (Hamilton et al., 1993), flufenamic acid (Qi et al., 2011) and 4-hydroxy-chalcone (Polsinelli et al., 2016). To characterize the role of K15 in defining TTR binding towards PFASs, we compared the binding affinities of PFASs towards the wild-type TTR and TTRmutK15G mutant. As shown in Table 1 , the $K_{\mathrm{d}}$ value of T4 to TTRmutK15G were much higher (about 1500-fold) than that of wild-type TTR, which is in good agreement with the crystal result that K15 forms a crucial hydrogen bond interaction with $\mathrm{T} 4$. The results showed that the $K_{\mathrm{d}}$ values of ten perfluoroalkyl acids and three perfluoroalkyl sulfonates to TTRmutK15G were much higher (641-22,971-fold) than those of wild-type TTR, thus confirming the crucial role K15 plays in binding these PFASs.

The results of molecular docking well explained the role of the K15 residue in the binding of PFASs to TTR. PFASs with a carboxylate or sulfonate end group were found to bind to TTR with the charged end group forming a hydrogen bond with K15. For the three charged end groups, their abilities to form hydrogen bonds with lysine followed the order of sulfonate > carboxylate > alcoholic hydroxyl. This is in agreement with the results that the binding potencies of PFASs are dependent on the charged end group with the order of sulfonate $>$ carboxylate $>$ alcoholic hydroxyl. The binding and docking results suggest strongly that the hydrogen bond between the charged end group and K15 plays an important role in the interaction between PFASs and TTR. The docking results also showed that PFASs with different carbon chain lengths bound to TTR using different modes. For the short-chain perfluoroalkyl acids (4C-8C), the hydrophobic region of these compounds buried fully within the TTR ligand-binding pocket. The binding pocket of T4 in TTR is a hydrophobic channel (Hamilton and Benson, 2001) and we hypothesize that as the carbon number increases to $\mathrm{C} 8$, this increase in ligand hydrophobicity strengthens the interaction between the PFASs and TTR and leads to higher binding affinity. The PFOA (8C) could nearly fill the TTR ligandbinding pocket, thus had the highest binding affinity. However, for the long-chain perfluoroalkyl acids (9C-14C), their molecular sizes are larger than the volume of the T4 binding pocket. Thus, the longer perfluoroalkyl acids cannot adopt an extended state in the binding pocket. Here, hydrophobic regions form a turn to adopt conformations that fit into the binding pocket. This tighter fit might destabilize the binding between perfluoroalkyl acids and TTR. Our binding and docking results strongly suggest that PFASs with medium chain length (with 8 carbons) are optimal for TTR binding.

For the TBG, only PFTA and PFTdA were found to bind to TBG. This is different from the pattern of binding of PFASs to TTR. Similar results have been observed in the study of binding of OH-PBDEs with $\mathrm{TH}$ transport proteins. OH-PBDEs with medium size had the highest binding affinity to TTR, whereas the OH-PBDEs with the larger size are optimal for TBG binding (Cao et al., 2010; Ren and Guo, 2012). The different binding patterns of ligands to TTR and TBG might be due to their different patterns of T4 binding pocket, which is a hydrophobic channel in TTR but a surface pocket in TBG (Hamilton et al., 1993; Zhou et al., 2006). According to several TBG-ligands crystal results, both residues R378 and R381 of TBG play crucial roles in the interaction with ligands (Zhou et al., 2006; Zheng, in press). Here, we found mutation of R378 or R381 to glycine resulted in significantly weaker binding of these two TBG mutants towards PFTA and PFTdA when compared with the binding affinities of the wild-type protein (Table 2). Thus, both R378 and R381 of TBG play crucial roles in the binding of PFTA and PFTdA.

The molecular docking analysis was also used in an effort to understand the binding results between PFASs and TBG. Spacefilling models showed that the perfluoroalkyl acids with carbon chain longer than $12 \mathrm{C}$ could fill the TBG ligand-binding pocket, whereas the short-chain PFASs could not. Thus, we inferred that for PFASs with carbon chain lengths $<\mathrm{C} 12$, these molecules could only form a hydrogen bond with R381, which might be insufficient for these ligands to adopt a stable bound state. For PFASs with carbon chain lengths $>\mathrm{C} 12$, these compounds might form a more stable interaction because of both the hydrogen bond with R381 and the interaction between the hydrophobic end of the ligand and R378. However, we inferred that the binding mode of PFTA and PFTdA to TBG is not a very favorable state because the binding affinities of them to TBG were very weak (4000-fold weaker than T4). Overall, the docking results appear to explain our observed binding results well. The results could explain why only PFTA and PFTdA bound to TBG and the critical role of R378 and R381 in forming a complex with these two compounds.

\section{Conclusions}

In this study, the binding affinities of 16 PFASs to TTR and TBG were determined, and critical structural features of their interactions were identified. Using the obtained $K_{\mathrm{d}}$ values, it was estimated that displacement of T4 from TTR by PFASs would be significant for the occupationally exposed workers but not the general population. The results of a competitive binding assay revealed that binding affinities of TTR to PFASs were much stronger than the binding affinities of TBG to the same PFASs. Thus, PFASs might bind to TTR but not TBG when they are ingested and enter the blood stream. The structure-binding relationship results showed that the binding affinities of PFASs to TTR and TBG are clearly associated with the carbon chain length and the charge of the end group. PFASs with a length of C8 and a sulfonate end group were optimal for TTR binding, whereas PFASs with a length longer than $\mathrm{C} 12$ bound TBG with modest affinity. By combining protein mutagenesis and computational modeling, we showed that residue K15 might play a key role in the binding of PFASs to TTR by forming a hydrogen bond with the acid end groups. Residues 
R378 and R381 might play crucial roles in the binding of PFTA and PFTdA to TBG owing to their roles in forming a hydrogen bond and stabilizing the hydrophobic end, respectively. The results should improve our understanding of the molecular mechanism of the interaction of PFASs with TH transport proteins, and the behavior and toxic mode of action of PFASs.

\section{Conflict of interests}

The authors have no conflict of interests to declare.

\section{Acknowledgments}

This work was supported by the Chinese Academy of Sciences (XDB14040100) and the National Natural Science Foundation of China (Grant Nos. 21407168, 21321004, 21375143, 91543203, 21377142, and 21477146).

\section{Appendix A. Supplementary data}

Supplementary data associated with this article can be found, in the online version, at http://dx.doi.org/10.1016/j.tox.2016.08.011.

\section{References}

Berg, V., Nost, T.H., Hansen, S., Elverland, A., Veyhe, A.S., Jorde, R., Odland, J.O., Sandanger, T.M., 2015. Assessing the relationship between perfluoroalkyl substances, thyroid hormones and binding proteins in pregnant women; a longitudinal mixed effects approach. Environ. Int. 77, 63-69.

Brent, G.A., 2012. Mechanisms of thyroid hormone action. J. Clin. Invest. 122, 30353043.

Calafat, A.M., Wong, L.Y., Kuklenyik, Z., Reidy, J.A., Needham, L.L., 2007. Polyfluoroalkyl chemicals in the U.S. population: data from the National Health and Nutrition Examination Survey (NHANES) 2003-2004 and comparisons with NHANES 1999-2000. Environ. Health Perspect. 115, 1596-1602.

Cao, J., Lin, Y., Guo, L.H., Zhang, A.Q., Wei, Y., Yang, Y., 2010. Structure-based investigation on the binding interaction of hydroxylated polybrominated diphenyl ethers with thyroxine transport proteins. Toxicology 277, 20-28.

Chang, S.C., Thibodeaux, J.R., Eastvold, M.L., Ehresman, D.J., Bjork, J.A., Froehlich, J. W., Lau, C., Singh, R.J., Wallace, K.B., Butenhoff, J.L., 2008. Thyroid hormone status and pituitary function in adult rats given oral doses of perfluorooctane sulfonate (PFOS). Toxicology 243, 330-339.

Chen, Y.M., Guo, L.H., 2009. Fluorescence study on site-specific binding of perfluoroalkyl acids to human serum albumin. Arch. Toxicol. 83, 255-261.

Dallaire, R., Dewailly, E., Pereg, D., Dery, S., Ayotte, P., 2009. Thyroid function and plasma concentrations of polyhalogenated compounds in Inuit adults. Environ. Health Perspect. 117, 1380-1386.

EPA, 2011. 2010/2015 PFOA Stewardship Program. US EPA, Office of Pollution Prevention and Toxics. <http://www.epa.gov/oppt/pfoa/pubs/stewardship/ index.html>.

EPA, 2013. Regulatory Action on PFAS/LCPFAC Compounds. US EPA, Office of Pollution Prevention and Toxics. <http://www.epa.gov/oppt/pfoa/pubs/pfas. html\#proposed>.

Fromme, H., Tittlemier, S.A., Völkel, W., Wilhelm, M., Twardella, D., 2009. Perfluorinated compounds-exposure assessment for the general population in Western countries. Int. J. Hyg. Environ. Health 212, 239-270.

Gutshall, D.M., Pilcher, G.D., Langley, A.E., 1988. Effect of thyroxine supplementation on the response to perfluoro-n-decanoic acid (PFDA) in rats. J. Toxicol. Environ. Health 24, 491-498.

Gutshall, D.M., Pilcher, G.D., Langley, A.E., 1989. Mechanism of the serum thyroid hormone lowering effect of perfluoro-n-decanoic acid (PFDA) in rats. J. Toxicol. Environ. Health 28, 53-65.

Hamilton, J.A., Benson, M.D., 2001. Transthyretin: a review from a structural perspective. Cell. Mol. Life Sci. 58, 1491-1521.

Hamilton, J.A., Steinrauf, L.K., Braden, B.C., Liepnieks, J., Benson, M.D., Holmgren, G, Sandgren, O., Steen, L., 1993. The X-ray crystal structure refinements of norma human transthyretin and the amyloidogenic Val-30- > Met variant to 1.7-A resolution. J. Biol. Chem. 268, 2416-2424.

Han, X., Snow, T.A., Kemper, R.A., Jepson, G.W., 2003. Binding of perfluorooctanoic acid to rat and human plasma proteins. Chem. Res. Toxicol. 16, 775-781.

Jones, P.D., Hu, W., De Coen, W., Newsted, J.L., Giesy, J.P., 2003. Binding of perfluorinated fatty acids to serum proteins. Environ. Toxicol. Chem. 22, 26392649.

Kato, K., Wong, L.Y., Jia, L.T., Kuklenyik, Z., Calafat, A.M., 2011. Trends in exposure to polyfluoroalkyl chemicals in the U.S. Population: 1999-2008. Environ. Sci. Technol. 45, 8037-8045.
Kovarich, S., Papa, E., Li, J., Gramatica, P., 2012. QSAR classification models for the screening of the endocrine-disrupting activity of perfluorinated compounds. SAR QSAR Environ. Res. 23, 207-220.

Langley, A.E., Pilcher, G.D., 1985. Thyroid, bradycardic and hypothermic effects of perfluoro-n-decanoic acid in rats. J. Toxiciol. Environ. Health 15, 485-491.

Lau, C., Thibodeaux, J.R., Hanson, R.G., Rogers, J.M., Grey, B.E., Stanton, M.E., Butenhoff, J.L., Stevenson, L.A., 2003. Exposure to perfluorooctane sulfonate during pregnancy in rat and mouse. II. Postnatal evaluation. Toxicol. Sci. 74 382-392.

Lau, C., Anitole, K., Hodes, C., Lai, D., Pfahles-Hutchens, A., Seed, J., 2007. Perfluoroalkyl acids: a review of monitoring and toxicological findings. Toxicol. Sci. 99, 366-394.

Lau, C., 2012a. Perfluorinated compounds. EXS 101, 47-86.

Lau, C., 2012b. Perfluoroalkyl acids: recent research highlights. Reprod. Toxicol. 33, 405-409.

Lindstrom, A.B., Strynar, M.J., Libelo, E.L., 2011. Polyfluorinated compounds: past, present, and future. Environ. Sci. Technol. 45, 7954-7961.

Liu, Y., Wang, J., Fang, X., Zhang, H., Dai, J., 2011. The thyroid-disrupting effects of long-term perfluorononanoate exposure on zebrafish (Danio rerio). Ecotoxicology 20, 47-55.

Liu, C., Wang, C., Yan, M., Quan, C., Zhou, J., Yang, K., 2012. PCB153 disrupts thyroid hormone homeostasis by affecting its biosynthesis, biotransformation, feedback regulation, and metabolism. Horm. Metab. Res. 44, 662-669.

Lopez-Espinosa, M.J., Mondal, D., Armstrong, B., Bloom, M.S., Fletcher, T., 2012. Thyroid function and perfluoroalkyl acids in children living near a chemical plant. Environ. Health Perspect. 120, 1036-1041.

Martin, J.W., Mabury, S.A., O'Brien, P.J., 2005. Metabolic products and pathways of fluorotelomer alcohols in isolated rat hepatocytes. Chem. Biol. Interact. 155, 165-180.

Melzer, D., Rice, N., Depledge, M.H., Henley, W.E., Galloway, T.S., 2010. Association between serum perfluorooctanoic acid (PFOA) and thyroid disease in the U.S. National Health and Nutrition Examination Survey. Environ. Health Perspect. 118, 686-692.

Neumann, P., Cody, V., Wojtczak, A., 2001. Structural basis of negative cooperativity in transthyretin. Acta Biochim. Pol. 48, 867-875.

Olsen, G.W., Zobel, L.R., 2007. Assessment of lipid, hepatic, and thyroid parameters with serum perfluorooctanoate (PFOA) concentrations in fluorochemical production workers. Int. Arch. Occup. Environ. Health 81, 231-246.

Olsen, G.W., Burris, J.M., Burlew, M.M., Mandel, J.H., 2003. Epidemiologic assessment of worker serum perfluorooctanesulfonate (PFOS) and perfluorooctanoate (PFOA) concentrations and medical surveillance examinations. J. Occup. Environ. Med. 45, 260-270.

Olsen, G.W., Burris, J.M., Ehresman, D.J., Froehlich, J.W., Seacat, A.M., Butenhoff, J.L., Zobel, L.R., 2007. Half-life of serum elimination of perfluorooctanesulfonate, perfluorohexanesulfonate, and perfluorooctanoate in retired fluorochemical production workers. Environ. Health Perspect. 115, 1298-1305.

Polsinelli, I., Nencetti, S., Shepard, W., Ciccone, L., Orlandini, E., Stura, E.A., 2016. A new crystal form of human transthyretin obtained with a curcumin derived ligand. J. Struct. Biol. 194, 8-17.

Qi, X., Loiseau, F., Chan, W.L., Yan, Y., Wei, Z., Milroy, L.G., Myers, R.M., Ley, S.V., Read, R.J., Carrell, R.W., Zhou, A., 2011. Allosteric modulation of hormone release from thyroxine and corticosteroid-binding globulins. J. Biol. Chem. 286, 16163-16173.

Ren, X.M., Guo, L.H., 2012. Assessment of the binding of hydroxylated polybrominated diphenyl ethers to thyroid hormone transport proteins using a site-specific fluorescence probe. Environ. Sci. Technol. 46, 4633-4640.

Ren, X.M., Zhang, Y.F., Guo, L.H., Qin, Z.F., Lv, Q.Y., Zhang, L.Y., 2015. Structure-activity relations in binding of perfluoroalkyl compounds to human thyroid hormone T3 receptor. Arch. Toxicol. 89, 233-242.

Schüttelkopf, A.W., van Aalten, D.M., 2004. PRODRG: a tool for high-throughput crystallography of protein-ligand complexes. Acta Crystallogr. D Biol. Crystallogr. D60, 1355-1363.

Schreiber, G., 2002. The evolutionary and integrative roles of transthyretin in thyroid hormone homeostasis. J. Endocrinol. 175, 61-73.

Seacat, A.M., Thomford, P.J., Hansen, K.J., Clemen, L.A., Eldridge, S.R., Elcombe, C.R., Butenhoff, J.L., 2003. Sub-chronic dietary toxicity of potassium perfluorooctanesulfonate in rats. Toxicology 183, 117-131.

Shenkin, A., 2006. Serum prealbumin: is it a marker of nutritional status or of risk of malnutrition? Clin. Chem. 52, 2177-2179.

Smith, D.S., 1977. Enhancement fluoroimmunoassay of thyroxine. FEBS Lett. 77, 2527.

Thibodeaux, J.R., Hanson, R.G., Rogers, J.M., Grey, B.E., Barbee, B.D., Richards, J.H., Butenhoff, J.L., Stevenson, L.A., Lau, C., 2003. Exposure to perfluorooctane sulfonate during pregnancy in rat and mouse. I. Maternal and prenatal evaluations. Toxicol. Sci. 74, 369-381.

Ucan-Marin, F., Arukwe, A., Mortensen, A.S., Gabrielsen, G.W., Letcher, R.J., 2010 Recombinant albumin and transthyretin transport proteins from two gull species and human: chlorinated and brominated contaminant binding and thyroid hormones. Environ. Sci. Technol. 44, 497-504.

Webster, G.M., Venners, S.A., Mattman, A., Martin, J.W., 2014. Associations between perfluoroalkyl acids (PFASs) and maternal thyroid hormones in early pregnancy: a population-based cohort study. Environ. Res. 133, 338-347.

Weiss, J.M., Andersson, P.L., Lamoree, M.H., Leonards, P.E., van Leeuwen, S.P., Hamers, T., 2009. Competitive binding of poly- and perfluorinated compounds to the thyroid hormone transport protein transthyretin. Toxicol. Sci. 109, 206216. 
Wenzel, K.W., 1981. Pharmacological interference with in vitro tests of thyroid function. Metabolism 30, 717-732.

Wojtczak, A., Neumann, P., Cody, V., 2001. Structure of a new polymorphic monoclinic form of human transthyretin at 3 A resolution reveals a mixed complex between unliganded and T4-bound tetramers of TTR. Acta Crystallogr. D Biol. Crystallogr. 57, 957-967.

Yen, P.M., 2001. Physiological and molecular basis of thyroid hormone action. Physiol. Rev. 81, 1097-1142.
Yu, W.G., Liu, W., Jin, Y.H., 2009. Effects of perfluorooctane sulfonate on rat thyroid hormone biosynthesis and metabolism. Environ. Toxicol. Chem. 28, 990-996. Zheng, J., Hashimoto, A., Putnam, M., Miller, K., Koh, J.T., 2008. Development of a thyroid hormone receptor targeting conjugate. Bioconjug. Chem. 19,1227-1234.

Zheng, Y., Structural mechanism of hormone release in thyroxine Binding globulin (in press).

Zhou, A., Wei, Z., Read, R.J., Carrell, R.W., 2006. Structural mechanism for the carriage and release of thyroxine in the blood. Proc. Natl. Acad. Sci. 103, 13321-13326. 